PROCEEDINGS OF THE

AMERICAN MATHEMATICAL SOCIETY

Volume 129, Number 8, Pages 2245-2251

S 0002-9939(01)06078-6

Article electronically published on March 20, 2001

\title{
ITERATIVE APPROXIMATION OF FIXED POINTS OF LIPSCHITZ PSEUDOCONTRACTIVE MAPS
}

\author{
C. E. CHIDUME
}

(Communicated by Jonathan M. Borwein)

\begin{abstract}
Let $E$ be a $q$-uniformly smooth Banach space possessing a weakly sequentially continuous duality map (e.g., $\ell_{p}, 1<p<\infty$ ). Let $T$ be a Lipschitzian pseudocontractive selfmapping of a nonempty closed convex and bounded subset $K$ of $E$ and let $\omega \in K$ be arbitrary. Then the iteration sequence $\left\{z_{n}\right\}$ defined by $z_{0} \in K, \quad z_{n+1}=\left(1-\mu_{n+1}\right) \omega+\mu_{n+1} y_{n} ; \quad y_{n}=$ $\left(1-\alpha_{n}\right) z_{n}+\alpha_{n} T z_{n}$, converges strongly to a fixed point of $T$, provided that $\left\{\mu_{n}\right\}$ and $\left\{\alpha_{n}\right\}$ have certain properties. If $E$ is a Hilbert space, then $\left\{z_{n}\right\}$ converges strongly to the unique fixed point of $T$ closest to $\omega$.
\end{abstract}

\section{INTRODUCTION}

Suppose $E$ is an arbitrary real Banach space with dual $E^{*}$. Given a gauge function $\phi:[0, \infty) \mapsto[0, \infty)$ the mapping $J_{\phi}: E \mapsto 2^{E^{*}}$ defined by

$$
J_{\phi} x:=\left\{u^{*} \in E^{*}:\left\langle x, u^{*}\right\rangle=\|x\| .\left\|u^{*}\right\| ;\left\|u^{*}\right\|=\phi(\|x\|)\right\}
$$

is called the duality map with gauge function $\phi$. In the particular case $\phi(t)=t$, the duality map $J=J_{\phi}$ is called the normalized duality map. For $q>1$, let $\phi(t)=t^{q-1}$ be a gauge function. We define the generalized duality map $J_{q}: E \mapsto 2^{E^{*}}$ by

$$
J_{\phi(t)}(x):=J_{q}(x)=\left\{x^{*} \in E^{*}:\left\langle x, x^{*}\right\rangle=\|x\| .\left\|x^{*}\right\| ;\left\|x^{*}\right\|=\|x\|^{q-1}\right\},
$$

and observe that for $q=2$ we write $J_{q}=J_{2}=J$ (the normalized duality map). It is well known that if $E^{*}$ is strictly convex, then $J$ is single-valued. In the sequel, we shall denote the single-valued generalized duality map by $j_{p}$. Let $K$ be a nonempty subset of $E$ and $T: K \mapsto E$ any map. Then $T$ is called pseudocontractive if and only if for all $x, y \in K$ there exists $j(x-y) \in J(x-y)$ such that $\langle T x-$ $T y, j(x-y)\rangle \leq\|x-y\|^{2}$, and is called strongly pseudocontractive if and only if there exists $k \in[0,1)$ such that for all $x, y \in K$ there exists $j(x-y) \in J(x-y)$ with $\langle T x-T y, j(x-y)\rangle \leq k\|x-y\|^{2}$. The map $T$ is called nonexpansive if and only if $\|T x-T y\| \leq\|x-y\|$ for all $x, y \in K$.

Interest in pseudocontractive maps stems mainly from their firm connection with the important class of nonlinear accretive operators where a mapping $U$ with domain $D(U)$ and range $R(U)$ in $E$ is called accretive if the inequality

$$
\|x-y\| \leq\|x-y+s(U x-U y)\|
$$

Received by the editors September 27, 1999.

2000 Mathematics Subject Classification. Primary 47H09, 47J05, 47J25.

Key words and phrases. Pseudocontractive operators, q-uniformly smooth spaces, duality maps, weak sequential continuity. 
holds for every $x, y \in D(U)$ and for all $s>0$. It is now well known (see, e.g., [3]) that if $T$ is accretive, then the solutions of the equation $T x=0$ correspond to the equilibrium points of some evolution systems. Consequently, considerable research efforts, especially within the past 15 years or so, have been devoted to iterative methods for approximating solutions of $T u=0$ when $T$ is accretive (or correspondingly, fixed points of pseudocontractions). In the special case in which the operator is Lipschitz and strongly pseudocontractive (or correspondingly Lipschitz and strongly accretive), the Mann iteration process (see, e.g., [8]) has been successfully employed. Results which had been known only in Hilbert spaces and only for Lipschitz maps (thanks to several geometric inequalities for general Banach spaces developed within the past 15 years or so) have been extended to more general Banach spaces and to more general classes of maps (see, e.g., 2 for relevant references). As has been pointed out in 2, this success has not carried over to arbitrary Lipschitz pseudocontractions $T$ even when the domain of the operator $T$ is a compact convex subset of a Hilbert space. In fact, it is still an open question whether or not the Mann iteration process converges under this setting (see, e.g., [2], 6]). In 1974, Ishikawa [7 introduced an iteration process which, in some sense, is more general than that of Mann and which converges, under this setting, to a fixed point of $T$. He proved the following theorem.

Theorem 1.1 (Ishikawa, [7). If $K$ is a compact convex subset of a Hilbert space $H, T: K \mapsto K$ is a Lipschitzian pseudocontractive map and $x_{0}$ is any point of $K$, then the sequence $\left\{x_{n}\right\}_{n \geq 0}$ converges strongly to a fixed point of $T$, where $x_{n}$ is defined iteratively for each positive integer $n \geq 0$ by

$$
x_{n+1}=\left(1-\alpha_{n}\right) x_{n}+\alpha_{n} T y_{n} ; y_{n}=\left(1-\beta_{n}\right) x_{n}+\beta_{n} T x_{n},
$$

where $\left\{\alpha_{n}\right\},\left\{\beta_{n}\right\}$ are sequences of positive numbers satisfying the conditions (i) $0 \leq$ $\alpha_{n} \leq \beta_{n}<1$; (ii) $\lim _{n \rightarrow \infty} \beta_{n}=0$; (iii) $\sum_{n \geq 0} \alpha_{n} \beta_{n}=\infty$.

Since its publication in 1974, Theorem 1.1, as far as we know, has never been extended to more general Banach spaces. All extensions proved so far are to slightly more general classes of operators and are still confined to Hilbert spaces (see, e.g., [2], 10], 11]). Other iteration processes have also been introduced. In particular, Schu 14 introduced an iteration process with a recursion formula similar to (1) and proved that if $K$ is a nonempty closed convex and bounded subset of a Hilbert space and $T: K \mapsto K$ is pseudocontractive and Lipschitzian, then the sequence generated from the recursion formula converges strongly to the unique fixed point of $T$ of minimum norm. By a different approach, Bruck [1] found another iteration procedure which converges, still in Hilbert spaces, to the same fixed point of $T$. Both Schu [14] and Bruck [1] made essential use of ideas which are originally due to Halpern [5] who was concerned with fixed points of nonexpansive mappings.

It is our purpose in this paper to prove that the iteration process studied by Schu [14] converges strongly, to a fixed point of a pseudocontractive Lipschitz map in certain real Banach spaces more general than Hilbert spaces. No compactness assumption will be imposed on the domain of our operator. Theorems 3.1, 3.2 and Corollary 3.3 are the first convergence theorems for iterative methods for fixed points of Lipschitz pseudocontractions in Banach spaces more general than Hilbert spaces. 


\section{Preliminaries}

Let $E$ be a real normed linear space of dimension dim. $E \geq 2$. The modulus of smoothness of $E$ is defined by

$$
\rho_{E}(\tau):=\sup \left\{\frac{\|x+y\|+\|x-y\|}{2}-1:\|x\|=1,\|y\|=\tau\right\} ; \quad \tau>0 .
$$

If $\rho_{E}(\tau)>0 \forall \tau>0$, then $E$ is said to be smooth. If there exist a constant $c>0$ and a real number $1<q<\infty$, such that $\rho_{E}(\tau) \leq c \tau^{q}$, then $E$ is said to be $q$-uniformly smooth. Typical examples of such spaces are the Lebesque $L_{p}$, the sequence $\ell_{p}$ and the Sobolev $W_{p}^{m}$ spaces for $1<p<\infty$. In particular, for $1<p \leq 2$, these spaces are $p$-uniformly smooth and for $2 \leq p<\infty$, they are 2 -uniformly smooth (see, e.g., [15]).

If $E$ is a real $q$-uniformly smooth Banach space, then (see, e.g., 15]) the following geometric inequality holds:

$$
\|x+y\|^{q} \leq\|x\|^{q}+q\left\langle y, j_{q}(x)\right\rangle+C_{q}\|y\|^{q}
$$

for all $x, y \in E$ and some real constant $C_{q} \geq 1$.

In the sequel, $\rightarrow$ and $\rightarrow$ will denote strong and weak convergence, respectively. The set of fixed points $F(T)$ of an operator $T$ is defined as follows: $F(T):=\{x \in$ $E: T x=x\}$. In what follows, we shall make use of the following lemmas, due to Schu [14], whose proofs we sketch for completeness.

Lemma 2.1. Let $E$ be a normed space possessing a duality map $j: E \mapsto E^{*}$ which is weakly sequentially continuous. Let (i) $K$ be a nonempty closed subset of $E$; (ii) $T: K \mapsto K$ be continuous and pseudocontractive; (iii) $\lambda_{n} \in(0,1)$ be such that $\lim \lambda_{n}=1$; (iv) $\left\{x_{n}\right\} \subseteq K$ be such that $x_{n}=\lambda_{n} T x_{n}, \forall n \in N ; x_{n} \rightarrow x^{*}$ for some $x^{*} \in E$. Then (a) $x_{n} \rightarrow x^{*}$ and $T x^{*}=x^{*}$; (b) $\left\langle x_{n}, j\left(y-x_{n}\right)\right\rangle \geq 0 \quad \forall y \in F(T)$; (c) $\left\langle x^{*}, j\left(y-x^{*}\right)\right\rangle \geq 0 \quad \forall y \in F(T)$.

Proof. Since $E$ possesses a duality map that is weakly sequentially continuous, it is smooth (see, e.g., [4]). Then, part (a) follows from [9], Lemma 2.5 and the proof of [9], Lemma 2.7. For part (b), fix $n \in N, y \in F(T)$ and define $\alpha_{n}:=\lambda_{n}^{-1}-1>0$. Then, using the pseudocontractivity of $T$, we obtain the following estimate:

$$
\begin{aligned}
\left\langle\lambda_{n}^{-1} x_{n}-y, j\left(y-x_{n}\right)\right\rangle & =\left\langle T x_{n}-T y, j\left(y-x_{n}\right)\right\rangle \\
& \geq-\left\|x_{n}-y\right\|^{2}=\left\langle x_{n}-y, j\left(y-x_{n}\right)\right\rangle
\end{aligned}
$$

so that $\left\langle\alpha_{n} x_{n}, j\left(y-x_{n}\right)\right\rangle \geq 0$, which establishes (b) since $\alpha_{n}>0$. Since $x_{n} \rightarrow x^{*}$, it follows from part (b) and the assumption on $j$ that for each fixed $y \in F(T)$, $\left\langle x^{*}, j\left(y-x^{*}\right)\right\rangle=\lim \left\langle x_{n}, j\left(y-x_{n}\right)\right\rangle \geq 0$.

Lemma 2.2. Let $E$ be a reflexive Banach space possessing a weakly sequentially continuous duality map $J: E \mapsto E^{*}$. Let (i) $K$ be a nonempty closed convex and bounded subset of $E$, and $\omega$ be an arbitrary vector in $K$; (ii) $T: K \mapsto K$ be a continuous and pseudocontractive map; (iii) $\left\{\lambda_{n}\right\}$ be a sequence in $(0,1)$ such that $\lim \lambda_{n}=1$. Then, (a) For each $n \in N$ there exists exactly one $x_{n} \in K$ such that $x_{n}=\left(1-\lambda_{n}\right) \omega+\lambda_{n} T x_{n}$; (b) $\left\{x_{n}\right\}$ converges strongly to some fixed point of $T$.

Proof. Without loss of generality, we may assume $\omega=0$. For $n \in N, T_{n}:=\lambda_{n} T$ is clearly strongly pseudocontractive and since $0 \in K$ and $K$ is convex, it follows that $T_{n}$ maps $K$ into itself. Corollary 1 of [3] then yields that $T_{n}$ possesses exactly one fixed point $x_{n} \in K$, completing the proof of part (a). For part (b), since $E$ is 
reflexive and $\left\{x_{n}\right\}$ is bounded, Eberlein-Smul'yan theorem yields that there exists a subsequence $\left\{x_{n_{j}}\right\}$ of $\left\{x_{n}\right\}$ which converges weakly to some $x^{*} \in E$. By Lemma 2.1 applied to $\left\{x_{n_{j}}\right\}$, we obtain that $x^{*}$ is a fixed point of $T,\left\{x_{n_{j}}\right\}$ converges strongly to $x^{*}$ and $\left\langle x^{*}, j\left(y-x^{*}\right)\right\rangle \geq 0$ for all $y \in F(T)$.

Claim 1. $x^{*}$ is unique.

To see this, assume there exists $y^{*} \neq x^{*}$ such that $y^{*} \in F(T)$ and $\left\langle y^{*}, j\left(y-y^{*}\right)\right\rangle \geq$ $0 \forall y \in F(T)$. Substituting $x^{*}$ for $y$ in this inequality, $y^{*}$ for $y$ in $\left\langle x^{*}, j\left(y-x^{*}\right)\right\rangle \geq 0$ and adding the resulting inequalities lead to an obvious contradiction. Hence $\left\{x_{n}\right\}$ possesses a unique strong cluster point and thus $x_{n} \rightarrow x^{*}$ as $n \rightarrow \infty$.

Remark 2.3. It was shown implicitly in [1] that, in the case of $E=H$, a Hilbert space, there is exactly one fixed point $x^{*}$ of $T$ such that

$$
\left\|\omega-x^{*}\right\|=\inf _{u \in F(T)}\|u-\omega\|
$$

and $\left\{x_{n}\right\}$ converges strongly to $x^{*}$. To see this, we first note that by Lemma 2.2 , $F(T) \neq \emptyset$ and by Corollary 2.3 of $[12, F(T)$ is closed and convex. So, if $P: H \mapsto$ $F(T)$ denotes the proximity map of $H$ onto $F(T)$ (i.e., $\forall x \in H, P x=y \in F(T)$ where $\left.\|x-y\|=\inf _{u \in F(T)}\|x-u\|\right)$, then $x^{*}:=P(\omega)$ is clearly the unique fixed point of $T$ satisfying (3). To show that $\left\{x_{n}\right\}$ converges to $x^{*}$, assume, without loss of generality, that $\omega=0$ and set $s^{*}=\lim x_{n}$. Then, for each $y^{*} \in F(T)$, it follows from Lemma 2.1(c) that $\left\langle s^{*}, y^{*}-s^{*}\right\rangle \geq 0$ and so $\left\|s^{*}\right\| \leq\left\|y^{*}\right\|$. Hence, $s^{*}=x^{*}$.

Before we state our next lemma, we introduce the following definition which is partly due to Halpern [5].

Definition 2.4. Let $\alpha_{n} \in(0, \infty), \mu_{n} \in(0,1)$ for all nonnegative integers $n$. Then $\left(\left\{\alpha_{n}\right\},\left\{\mu_{n}\right\}\right)$ is said to have property $(A)$ if and only if the following conditions hold: (i) $\left\{\alpha_{n}\right\}$ is decreasing and $\left\{\mu_{n}\right\}$ is strictly increasing; (ii) there is a sequence $\left\{\beta_{n}\right\} \subset N$, strictly increasing, such that

$$
\begin{aligned}
& \text { (a) } \lim _{n \rightarrow \infty} \beta_{n}\left(1-\mu_{n}\right)=\infty ; \quad \text { (b) } \lim _{n \rightarrow \infty}\left(\frac{1-\mu_{n+\beta_{n}}}{1-\mu_{n}}\right)=1 ; \text { and } \\
& \text { (c) } \lim _{n \rightarrow \infty}\left(\frac{\alpha_{n}-\alpha_{n+\beta_{n}}}{1-\mu_{n}}\right)=0 .
\end{aligned}
$$

Lemma 2.5 (cf. [13]). Let $K$ be a nonempty convex and bounded subset of a normed linear space $E$ with $0 \in K$. Let (i) $S_{n}: K \mapsto K$ be such that $\| S_{n}(x)-$ $S_{n}(y)\left\|\leq k_{n}\right\| x-y \| \forall n \in N, \forall x, y \in K$ where $k_{n} \in[1, \infty)$; (ii) $\lambda_{n} \in(0,1), \alpha_{n} \in$ $(0, \infty)$ be such that $\left(\left\{\alpha_{n}\right\},\left\{\mu_{n}\right\}\right)$ has property $(A)$ and $\left(1-\mu_{n}\right)\left(1-\lambda_{n}\right)^{-1}$ is bounded, where $\mu_{n}:=\lambda_{n} k_{n}^{-1} \forall n \in N$; (iii) $\left\{x_{n}\right\} \subseteq K$ be such that $x_{n}=\mu_{n} S_{n}\left(x_{n}\right) \forall n \in N$ and there exists a constant $M>0$ such that $\left\|S_{i}(x)-S_{j}(x)\right\| \leq M\left|\alpha_{i}-\alpha_{j}\right| \forall i, j \in$ $N, \forall x \in K$. Assume that $\lim x_{n}:=x^{*}$ exists. Define the sequence $\left\{z_{n}\right\}$ by $z_{n+1}:=\mu_{n+1} S_{n}\left(z_{n}\right) \quad \forall n \in N$, where $z_{0} \in K$ is arbitrary. Then, $\lim _{n \rightarrow \infty} z_{n}=x^{*}$.

\section{MAIN RESULTS}

We prove the following theorems.

Theorem 3.1. Let $K$ be a nonempty subset of a q-uniformly smooth Banach space $E$ possessing a weakly sequentially continuous duality map. Suppose that (i) $T$ : $K \mapsto K$ is pseudocontractive and Lipschitzian (with Lipschitz constant $L \geq 0$ ); 
(ii) $\alpha_{n} \in(0, \infty), \mu_{n} \in(0,1), k_{n}:=\left[1+C_{q} \alpha_{n}^{q}(L+1)^{q}\right]^{\frac{1}{q}}$ and $S_{n}:=\left(1-\alpha_{n}\right) I+\alpha_{n} T$;

(iii) $\left\{x_{n}\right\} \subseteq K$ is such that for all positive integers $n$,

$$
x_{n}=\left[\frac{\mu_{n} \alpha_{n}}{1-\mu_{n}\left(1-\alpha_{n}\right)}\right] T x_{n} .
$$

Then, $\forall n \in N$, the following results hold:

$$
\text { (a) }\left\|S_{n}(x)-S_{n}(y)\right\| \leq k_{n}\|x-y\|, \quad \forall x, y \in K ; \quad \text { and } \quad \text { (b) } x_{n}=\mu_{n} S_{n}\left(x_{n}\right) .
$$

Proof. Define $G:=(I-T)$ and observe that for all $x, y \in K,\left\langle G x-G y, j_{q}(x-y)\right\rangle \geq 0$. Moreover, $S_{n}=I-\alpha_{n} G$ so that for all $x, y \in K$,

$$
\begin{aligned}
\left\|S_{n}(x)-S_{n}(y)\right\|^{q} \leq & \|x-y\|^{q}-q \alpha_{n}\left\langle G x-G y, j_{q}(x-y)\right\rangle \\
& +C_{q} \alpha_{n}^{q}(L+1)^{q}\|x-y\|^{q}, \\
\leq & {\left[1+C_{q} \alpha_{n}^{q}(L+1)^{q}\right]\|x-y\|^{q}, }
\end{aligned}
$$

since $\|G x-G y\| \leq(L+1)\|x-y\|$, and (a) follows. (b) is obvious.

Theorem 3.2. Let $K$ be a nonempty closed convex and bounded subset of a $q$ uniformly smooth Banach space possessing a weakly sequentially continuous duality map. Suppose that (i) $T: K \mapsto K$ is pseudocontractive and Lipschitzian (with Lipschitz constant $L \geq 0)$; (ii) $\lambda_{n} \in(0,1)$ with $\lim \lambda_{n}=1 ; \quad \alpha_{n} \in(0,1)$ with $\lim \alpha_{n}=0 ;\left(\left\{\alpha_{n}\right\},\left\{\mu_{n}\right\}\right)$ has property $(A) ;\left(1-\mu_{n}\right)\left(1-\lambda_{n}\right)^{-1}$ is bounded and $\lim \alpha_{n}^{-1}\left(1-\mu_{n}\right)=0$, where $\mu_{n}:=\lambda_{n} k_{n}^{-1}$ and $k_{n}:=\left[1+C_{q} \alpha_{n}^{q}(L+1)^{q}\right]^{q^{-1}}$. Fix $z_{0} \in K$ arbitrary and for each positive integer $n$, define the sequence $\left\{z_{n}\right\}$, for arbitrary $\omega \in K$, by

$$
z_{n+1}=\left(1-\mu_{n+1}\right) \omega+\mu_{n+1} y_{n} ; \quad y_{n}=\left(1-\alpha_{n}\right) z_{n}+\alpha_{n} T z_{n} .
$$

Then, $\left\{z_{n}\right\}$ converges strongly to a fixed point of $T$.

Proof. Without loss of generality, we may assume $\omega=0$. For any positive integer $n$, define

$$
\gamma_{n}:=\left[1+\frac{1}{\mu_{n}}\left(\frac{1-\mu_{n}}{\alpha_{n}}\right)\right]^{-1}
$$

Clearly, $\gamma_{n} \in(0,1)$. Since $\lim \mu_{n}=1$ and $\lim \alpha_{n}^{-1}\left(1-\mu_{n}\right)=0$ it follows that $\lim \gamma_{n}=1$. Hence, by Lemma 2.2, for each positive integer $n$, there exists a unique $x_{n} \in K$ such that $x_{n}=\gamma_{n} T x_{n}$ and $x_{n} \rightarrow x^{*}$, for some $x^{*} \in F(T)$. Furthermore, since $\alpha_{n} \in(0,1)$ we easily obtain that $S_{n}:=\left(1-\alpha_{n}\right) I+\alpha_{n} T$ maps $K$ into itself for each $n \in N$. Consequently, by Theorem 3.1, for each $n \in N, x_{n}=\mu_{n} S_{n}\left(x_{n}\right)$ and $S_{n}$ is Lipschitzian with constant $k_{n}$. Moreover, $z_{n+1}=\mu_{n+1} S_{n}\left(z_{n}\right)$ for all positive integers $n$, and $\left\|S_{i}(x)-S_{j}(x)\right\| \leq\left|\alpha_{i}-\alpha_{j}\right|$.diam. $(K)$ for all $i, j \in N$, all $x \in K$. It then follows from Lemma 2.5 that $z_{n} \rightarrow x^{*}$ as $n \rightarrow \infty$. This completes the proof.

Corollary 3.3. Let $K$ be a nonempty closed convex and bounded subset of $\ell_{p}, 1<$ $p<\infty$. Suppose $T: K \mapsto K$ is pseudocontractive and Lipschitzian (with Lipschitz constant $L \geq 0)$. Suppose further that $\omega, \lambda_{n}, \alpha_{n}, \mu_{n}$ and $k_{n}$ are as in (ii) of Theorem 3.2. Then, for arbitrary $z_{0} \in K$, the sequence $\left\{z_{n}\right\}$ defined by (4) converges strongly to a fixed point of $T$. 
Proof. The Banach space $\ell_{p}, \quad 1<p<\infty$, is $q$-uniformly smooth and possesses a weakly sequentially continuous duality map. The result follows from Theorem 3.2 .

As immediate consequences of Corollary 3.3, we obtain Corollaries 3.4 and 3.5 below which are the main results of Schu [14.

Corollary 3.4. Let $H$ be a Hilbert space and let (i) $K$ be a nonempty closed convex and bounded subset of $H, \omega \in K$ arbitrary; (ii) $T: K \mapsto K$ be pseudocontractive and Lipschitzian with constant $L \geq 0$; (iii) $\lambda_{n} \in(0,1)$ with $\lim \lambda_{n}=1 ; \alpha_{n} \in(0,1)$ with $\lim \alpha_{n}=0$ such that $\left(\left\{\alpha_{n}\right\},\left\{\mu_{n}\right\}\right)$ has property $(A),\left(1-\mu_{n}\right)\left(1-\lambda_{n}\right)^{-1}$ is bounded and $\lim \alpha_{n}^{-1}\left(1-\mu_{n}\right)=0$, where $k_{n}:=\left(1+\alpha_{n}^{2}(L+1)^{2}\right)^{\frac{1}{2}}$ and $\mu_{n}:=\lambda_{n} k_{n}^{-1} \forall n \in N$. Fix $z_{0} \in K$ arbitrary and define $\left\{z_{n}\right\}$ by (4) $\forall n \in N$. Then $\left\{z_{n}\right\}$ converges strongly to the unique fixed point of $T$ closest to $\omega$.

Proof. Set $p=2$ in Corollary 3.3. The result follows from Corollary 3.3 and Remark 2.3 .

Corollary 3.5. Let $H$ be a Hilbert space, and let (i) $K$ be a nonempty closed convex and bounded subset of $H, \omega \in K$ arbitrary; (ii) $T: K \mapsto K$ be pseudocontractive and Lipschitzian with constant $L \geq 0, z_{0} \in K$ arbitrary; (iii) For $n \in N$, set $\mu_{n}:=1-(n+1)^{\frac{-1}{2}}, \alpha_{n}:=(n+1)^{\frac{-1}{4}}(L+1)^{-1}$ and define $\left\{z_{n}\right\}$ by (4). Then $\left\{z_{n}\right\}$ converges strongly to the unique fixed point of $T$ closest to $\omega$.

Proof. For each $n \in N$, define $k_{n}:=\left(1+\alpha_{n}^{2}(L+1)^{2}\right)^{\frac{1}{2}}, \lambda_{n}:=\mu_{n} k_{n}$ and $\beta_{n}=$ $\left[(n+1)^{\frac{5}{8}}\right]$, where $[t]:=\max \{n \in N: n \leq t\}$. Then, it has been shown in [5] that conditions (iii), (a) and (b) of Definition 2.4 are already satisfied. Easy calculations show that all the other assumptions of Corollary 3.3 are satisfied. The result follows from Corollary 3.3.

Remark 3.6. Corollary 3.4 is, in several respects, an improvement on the theorem of Ishikawa [7]. In particular, the Corollary does not require that the subset $K$ be compact as is the case in [7]. Moreover, since the fixed points of a Lipschitz pseudocontraction are generally not unique, Corollary 3.4 gives the additional information (unlike in 7]) as to which fixed point the sequence converges. However, in the theorem of Ishikawa [7], the parameters of the recursion formula $\alpha_{n}, \beta_{n}$ can easily be chosen (e.g., $\alpha_{n} \equiv \frac{1}{\sqrt{n+1}} \equiv \beta_{n}$ ) whereas, as is easily seen from Corollary 3.5 , the choices of the iteration parameters $\alpha_{n}$ and $\mu_{n}$ are not so simple.

Remark 3.7. As has been noted in the introduction, Theorems 3.1, 3.2 and Corollary 3.3 are the first theorems on iterative methods for fixed points of Lipschitz pseudocontractions in Banach spaces more general than Hilbert spaces.

\section{Note ADDED IN PROOF}

The open question referred to in the introduction has recently been resolved: C. E. Chidume and S. Mutangadura, An example on the Mann iteration method for Lipschitz pseudocontractions, Proc. Amer. Math. Soc. (to appear), published electronically January 18, 2001. In this reference, an example of a Lipschitz pseudocontractive map with a unique fixed point is constructed for which the Mann iteration process fails to converge. 


\section{REFERENCES}

[1] R. E. Bruck, Jr.; A strongly convergent iterative method for the solution of $0 \in U x$ for a maximal monotone operator $U$ in Hilbert space, J. Math. Anal. Appl. 48 (1974), 114-126. MR 50:14383

[2] C. E. Chidume and C. Moore; Fixed point iteration for pseudocontractive maps, Proc. Amer. Math. Soc. 127(4) (1999), 1163-1170. MR 99f:47068

[3] K. Deimling; Zeros of accretive operators, Manuscripta Math. 13 (1974), 365-374. MR 50:3030

[4] J. Diestel; Geometry of Banach spaces - selected topics, Lecture Notes in Math. 485, Springer-Verlag, Berlin, Heidelberg, New York, 1976. MR 57:1079

[5] B. Halpern; Fixed points of nonexpansive maps, Bull. Amer. Math. Soc. 3 (1967), 957-961. MR 36:2022

[6] T. L. Hicks and J. R. Kubicek; On the Mann iteration process in Hilbert space, J. Math. Anal. Appl. 59 (1979), 498-504. MR 58:23802

[7] S. Ishikawa; Fixed points by a new iteration method, Proc. Amer. Math. Soc. 44(1) (1974), 147-150. MR 49:1243

[8] W. R. Mann; Mean value methods in iteration, Proc. Amer. Math. Soc. 4 (1953), 506-510. MR 14:988f

[9] G. Müller and J. Reinermann; Fixed point theorems for pseudocontractive mappings and a counter-example for compact maps, Comment. Math. Univ. Carolin. 18(2) (1977), 281-298. MR 56:6482

[10] Liu Qihou; On Naimpally and Singh's open question, J. Math. Anal. Appl. 124 (1987), 157164. MR 88j: 47078

[11] L. Qihou; The convergence theorems of the sequence of Ishikawa iterates for hemicontractive mappings, J. Math. Anal. Appl. 148 (1990), 55-62. MR 92b:47094

[12] R. Schöneberg; On the structure of fixed point sets of pseudocontractive mappings II, Comment. Math. Univ. Carolin. 18(2) (1977), 299-310. MR 58:23809

[13] J. Schu; Approximation of fixed points of asymptotically nonexpansive mappings, Proc. Amer. Math. Soc. 112(1) (1991), 143-151. MR 91h:47063

[14] J. Schu; Approximating fixed points of Lipschitz pseudocontractive mappings, Houston J. Math. 19 (1993), 107-115. MR 94f:47066

[15] Z. B. Xu and G. F. Roach; Characteristic inequalities for uniformly convex and uniformly smooth Banach spaces, J. Math. Anal. Appl. 157 (1991), 189-210. MR 92i:46023

The Abdus Salam International Centre for Theoretical Physics, P.O. Box 586, TriESTE, ITALY

E-mail address: chidume@ictp.trieste.it 